\title{
Percevoir son corps à travers le regard d'autrui : Une revue de la littérature sur l'auto-objectification
}

\author{
Robin Wollast ${ }^{* 1}$, Matthias De Wilde ${ }^{2}$, Philippe Bernard ${ }^{1}$ \\ et Olivier Klein ${ }^{1}$ \\ 1 Centre de recherche en psychologie sociale et interculturelle, \\ université libre de Bruxelles \\ 2 Centre pour l'étude du comportement social, université catholique de Louvain
}

\begin{abstract}
RÉSUMÉ
L'objectification sexuelle, à savoir la tendance à considérer ou à traiter une personne comme un objet sexuel, est présente au quotidien dans les relations interpersonnelles comme dans les médias. En effet, ceuxci (télévision, magazines, internet, jeux vidéo...) véhiculent des images sexualisées, idéalisées et stéréotypées des femmes et des hommes qui ne sont pas sans conséquence pour le grand public. Actuellement, de nombreuses études mettent en évidence que l'impossibilité pour une personne de ressembler ou de s'identifier à une construction inatteignable ou à un modèle parfait peut avoir des conséquences négatives comme de la honte corporelle, de l'anxiété par rapport à son apparence, des troubles alimentaires ou encore de la dépression. L'objectif de cette revue de la littérature est triple. Primo, elle introduit le lecteur à la théorie de l'objectification, développée par Fredrickson et Roberts (1997), qui décrit l'influence de cette représentation omniprésente et idéalisée de la beauté sur la santé mentale des femmes. Secundo, cette littérature centrée sur le concept d'auto-objectification présente de manière synthétique et structurée les études phares qui ont significativement contribué au développement de la réflexion sur la manière dont les hommes et les femmes perçoivent leur propre corps. Tertio, ce manuscrit décrit les limites actuelles de cette théorie et présente de nouvelles perspectives de recherche.
\end{abstract}

* Auteur correspondant : Robin Wollast, Centre de recherche en Psychologie sociale et interculturelle, Faculté des sciences psychologiques et de l'éducation, Université libre de Bruxelles, avenue F. D. Roosevelt, 50, Campus du Solbosch, Bruxelles, Belgique. Email : robin.wollast@ulb.ac.be 
Mots-clés : image du corps; théorie de l'objectification ; objectification sexuelle ; auto-objectification ; autosurveillance ; honte corporelle.

\section{Perceiving one's body through the eyes of others: A review of literature on self-objectification}

\section{ABSTRACT}

Sexual objectification (i.e., the treatment of a person as a sexual object) is now a part of individuals' daily interpersonal interactions and is highly apparent in the media (television, internet, music, magazines, video games). Indeed, the media convey sexualized, idealized and stereotyped images of women and men that deeply influence the public's attitudes towards their own bodies. According to objectification theory, being treated as an object leads women to engage in self-objectification, which in turns generates body shame and body surveillance as well as impairing their mental health (e.g., eating disorders, sexual dysfunction, depression). The purpose of this review of literature is threefold. First, we aim to introduce objectification theory, developed by Fredrickson and Roberts (1997), describing the influence of these ubiquitous representations of beauty on women's mental health. Second, we aim to present a structured synthesis of the prominent studies that have significantly contributed to the understanding of the objectification phenomenon. Third, we will describe the current limitations of this theory and propose new research perspectives.

Keywords: body image; objectification theory; sexual objectification; selfobjectification; body surveillance; body shame.

«Elle devient un objet; et elle se saisit comme objet ; c'est avec surprise qu'elle découvre ce nouvel aspect de son être : il lui semble qu'elle se dédouble; au lieu de coïncider exactement avec soi, voilà qu'elle se met à exister dehors " (Beauvoir, 1949, vol. II, p. 90).

\section{INTRODUCTION}

Depuis la nuit des temps, les femmes ont principalement été jugées sur leur apparence, en étant soumises à de nombreuses normes de beauté 
exigeantes, codifiées par la société (Vigarello, 2004). Pour les femmes, se conformer à des critères esthétiques nécessite parfois des transformations ou même des mutilations corporelles. Par exemple, en Chine, jusqu'au $\mathrm{XX}^{\mathrm{e}}$ siècle, une tradition millénaire voulait que les femmes se bandent les pieds pour les empêcher de grandir parce que cette atrophie était le symbole ultime de la féminité. Dans d'autres lieux, à d'autres époques, elles portent des corsets baleinés suffoquant ou de lourds colliers-spirales, se font tatouer le corps, s'astreignent à des régimes alimentaires stricts ou subissent de la chirurgie esthétique. Selon la théorie de l'objectification (Fredrickson \& Roberts, 1997), si les femmes s'imposent toutes ces contraintes, c'est en grande partie parce que leur contexte social les pousse à croire que leur corps est un objet sexuel à disposition des hommes. Ces pratiques sont la conséquence d'une représentation de la femme réduite au statut d'objet sexuel passif. Cette représentation, fortement répandue, est notamment véhiculée par les médias. Cette thématique a été amplement étudiée par les théoriciens et théoriciennes du féminisme (p. ex., Bauer, 2011 ; Bordo, 1993, 1999 ; Dworkin, 1985 ; Green, 2000 ; Haslanger, 2012; MacKinnon, 1987, 1989; Saul, 2003).

Le présent article a pour objectif premier d'introduire le lecteur à la théorie de l'objectification (Fredrickson \& Roberts, 1997), qui décrit l'influence de cette représentation omniprésente et idéalisée de la beauté féminine sur la santé mentale des femmes. Cette théorie, bien que fort influente dans le monde anglo-saxon et largement étayée empiriquement, n'a pas encore fait l'objet d'une présentation en langue française. Notre second objectif est de présenter de manière synthétique et structurée les travaux menés sur le concept d'auto-objectification, un concept qui a permis d'approfondir et d'enrichir notre compréhension de la manière dont les hommes et les femmes perçoivent leur propre corps. Pour ce faire, nous aborderons d'abord les facteurs responsables de ce phénomène d'objectification du corps. Nous en soulignerons ensuite les conséquences sur la santé mentale des individus et, enfin, les mécanismes explicatifs qui sous-tendent ce processus. Le troisième objectif est de discuter des limites actuelles de cette théorie et de proposer de nouvelles perspectives de recherche qui restent à explorer.

\section{HISTORIQUE DE L'OBJECTIFICATION}

Pour Kant (1797/1993), le phénomène d'objectification est commun dans toute relation sexuelle : il est présent dès lors qu'on envisage l'autre 
uniquement comme une façon de répondre à un «appétit sexuel ». Selon lui, l'objectification se caractérise principalement par la dimension instrumentale de la relation sexuelle entre un homme et une femme. En d'autres mots, l'objectification est le résultat d'une rupture entre le corps et l'âme. Par exemple, les relations extra-conjugales ou les rapports sexuels monnayés impliqueraient un rapport à l'autre centré uniquement sur son corps. En outre, dans des situations d'objectification, ce qui rend l'individu objectifié essentiellement humain aux yeux de Kant (sa rationalité, son libre-arbitre) est négligé et n'est, au mieux, qu'un moyen pour parvenir à combler des désirs sexuels (pour une analyse approfondie, voir Papadaki, 2007, 2010).

Actuellement, la définition la plus générale et consensuelle du concept d'objectification correspond au fait de traiter quelqu'un comme un objet (Papadaki, 2010). Derrière la simplicité apparente de cette définition se dissimule une réalité plus complexe. En effet, Martha Nussbaum (1995) suggère qu'il existe différentes manières de "traiter quelqu'un comme un objet " (instrumentalisation, déni d'autonomie, de subjectivité, de la capacité d'agir, interchangeabilité et autorisation morale d'être propriétaire ou de violer l'intégrité physique d'une personne; voir LaCroix \& Pratto [2015] pour une taxonomie plus détaillée). Par la suite, Langton (2009) complètera cette liste en ajoutant trois éléments supplémentaires, dont la réduction au corps, la réduction à l'apparence et la réduction au silence.

En somme, le processus d'objectification s'installe lorsque « les parties ou les fonctions du corps d'une personne sont séparées et réduites à un statut d'instrument ou si elles sont vues comme capables de représenter la personne dans son entièreté » (Bartky, 1990, p. 175). Depuis longtemps, ce phénomène d'objectification sexuelle a fait l'objet de nombreuses discussions en psychologie (Horney, 1967), en philosophie (Kant, 1797/ 1993), en histoire (Berger, 1972) ou encore en sociologie et économie (Marx, 1844/2007).

En psychologie sociale, la théorie de l'objectification développée par Fredrickson et Roberts (1997) permet d'expliquer l'impact négatif de l'objectification sur la santé mentale des femmes. Dans la section suivante, nous explicitons cette théorie en nous focalisant principalement sur un concept phare appelé l'auto-objectification. 


\section{OBJECTIFICATION SEXUELLE ET AUTO-OBJECTIFICATION}

Selon Fredrickson et Roberts (1997), l'expérience récurrente de comportements objectifiants (regards concupiscents, commentaires sexistes, allusions sexuelles, harcèlement de rue), ainsi que l'exposition répétée à des images de femmes hypersexualisées et attirantes (Ward, 2016) conduisent les femmes à intérioriser l'injonction implicite qui les encourage à considérer leur corps comme un objet qui peut être regardé et évalué par autrui. Ces expériences d'objectification sexuelle engendrent l'intériorisation du regard objectifiant porté par la société sur le corps des femmes. Ce phénomène porte le nom $d$ ' «auto-objectification ». En effet, la théorie de l'objectification postule que la culture (p. ex., rôles de genres traditionnels, princesses de Walt Disney), l'éducation (p. ex. les manuels scolaires) et les médias (p. ex., réseaux sociaux, publicité, télé-réalité, pornographie, clips ou paroles de chansons) véhiculent l'idée selon laquelle l'apparence physique d'une femme revêt une importance considérable par rapport aux autres aspects de sa personne. Dans ce contexte, l'image du corps de la femme correspond à celle d'un objet sexuel qui peut être instrumentalisé et évalué par autrui et qui doit correspondre à des standards de beauté irréalistes et inaccessibles pour la plupart des femmes ( $\mathrm{p}$. ex. la minceur).

L'auto-objectification se caractérise par une tendance à accorder davantage d'importance aux aspects physiques observables (p. ex., mensurations, poids) de sa personne qu'aux aspects physiques non observables (p. ex., santé, souplesse), impliquant des questions centrées sur l'apparence (« De quoi ai-je l'air ? ») plutôt que sur les capacités physiques (« De quoi suis-je capable?»). En somme, les femmes peuvent être amenées à considérer elles-mêmes leur corps comme un objet qui peut être regardé, évalué, jugé, désiré ou détesté par les autres (pour une revue de la littérature, voir Szymanski, Moffitt, \& Carr, 2011).

Fredrickson et Roberts (1997) postulent que l'auto-objectification peut prendre deux formes. Premièrement, elle peut être considérée comme une disposition stable, un trait, lorsqu'elle est profondément intériorisée. C'est ce dont témoigne l'existence de différences individuelles quant à la façon de percevoir son apparence physique. Deuxièmement, ces mêmes chercheuses suggèrent que certains contextes favorisent l'émergence de l'autoobjectification (p. ex., lieux publics, soirée dansante, plage). On parle dès 
lors d'auto-objectification " état ». De nombreuses études ont mis en évidence les différents contextes induisant un état transitoire d'auto-objectification. Par exemple, l'exposition à des remarques à caractère sexuel dans un lieu public (Swim, Hyers, Cohen, \& Ferguson, 2001), l'essayage d'un maillot de bain devant un miroir (Fredrickson, Roberts, Noll, Quinn, \& Twenge, 1998 ; Quinn, Kallen, \& Cathey, 2006; Tiggemann \& Boundy, 2008), la présence ostensible d'une caméra (Gay \& Castano, 2010 ; Saguy, Quinn, Dovidio, \& Pratto, 2010) et l'exposition à des images de femmes dénudées (Aubrey, 2006 ; Krawczyk \& Thompson, 2015) sont autant de contextes qui suscitent une baisse de l'estime de soi chez des femmes ainsi qu'une augmentation du sentiment d'auto-objectification. En règle générale, les situations dans lesquelles la présence d'un regard extérieur porté sur son corps est rendue saillante induiraient un tel sentiment (pour une revue de la littérature sur l'auto-objectification d'état, voir Kahalon, Shnabel, \& Becker, 2018).

Comme nous venons de le voir, les expériences d'objectification font partie du quotidien de la majorité des femmes. Selon la théorie de l'objectification, le caractère répétitif de ces expériences pousse certaines femmes à intérioriser le regard qu'autrui porte sur leur corps, à s'auto-objectifier de manière plus stable (Fredrickson \& Roberts, 1997). En d'autres termes, lorsque l'auto-objectification devient une disposition stable, la préoccupation qu'une femme entretient envers son apparence deviendra omniprésente, même en l'absence physique du regard d'autrui.

\section{LES CONSÉQUENCES NÉGATIVES DE L'AUTO-OBJECTIFICATION}

Indépendamment des travaux portés par la théorie de l'objectification, McKinley et Hyde (1996) décrivent les mécanismes sous-jacents liés à l'auto-objectification chez les femmes uniquement. Ces chercheuses proposent trois dimensions qui s'intéressent aux effets que peut engendrer l'intériorisation des standards de beauté : l'autosurveillance, la honte corporelle et le sentiment de contrôle. La première correspond au fait de s'engager dans des processus d'autosurveillance de leur propre corps, perçu comme un objet, en tant qu'observatrices extérieures capables de juger leur apparence en se comparant à la norme de beauté, mais aussi dans leurs relations interpersonnelles (p. ex. avec les pairs). La seconde 
dimension correspond à la propension qu'ont les femmes qui surveillent leur propre corps à ressentir de la honte corporelle par rapport à la difficulté de correspondre à des standards de beauté irréalistes établis par la société. Enfin, la troisième correspond au degré auquel les femmes pensent qu'elles peuvent contrôler leur image corporelle. Ce sentiment de contrôle peut les amener à se sentir responsables de la manière dont elles perçoivent leur corps. De fait, ce sentiment peut contribuer à atténuer le stress que peut susciter la comparaison aux des normes de beauté, mais peut également pousser les femmes à se montrer plus critiques par rapport à elles-mêmes lorsqu'elles ne sont pas satisfaites de leurs efforts. En ce sens, la littérature actuelle considère l'autosurveillance comme l'une des manifestations comportementales principales liées à l'auto-objectification (McKinley, 1999).

Par la suite, Fredrickson et Roberts (1997) mirent en évidence plusieurs conséquences délétères de cette auto-objectification et autosurveillance du corps (voir Figure 1) comme un sentiment de honte par rapport à son propre corps ou une augmentation de l'angoisse liée à son apparence physique (McKinley \& Hyde, 1996). De nombreuses autres études (Greenleaf, 2005 ; Muehlenkamp \& Saris-Baglama, 2002 ; Strelan, Mehaffey, \& Tiggemann, 2003) ont ensuite montré que l'auto-objectification génère des conséquences psychologiques dommageables telles qu'une baisse de l'estime de soi, une perturbation des états motivationnels, une perception plus faible des sensations internes, un affaiblissement de la santé physique et des troubles de santé mentale, comme des troubles alimentaires, des troubles dépressifs ou encore des troubles sexuels (pour des revues de la littérature, voir Moradi \& Huang, 2008 ; Roberts, Calogero, \& Gervais, 2017).

Dans ce même contexte, certains travaux avancent que la surveillance corporelle est consommatrice de ressources cognitives, ce qui peut avoir pour conséquence, une mobilisation plus faible des ressources attentionnelles dans l'exécution d'autres tâches (p. ex., tests cognitifs, prise de parole en public, sports). Par exemple, plusieurs études démontrent qu'un contexte d'objectification induit une diminution des performances cognitives (test de mathématiques; Fredrickson, Roberts, Noll, Quinn, \& Twenge, 1998 ; tâche de Stroop ; Quinn, Kallen, Twenge, \& Fredrickson, 2006), ainsi qu'une diminution de la satisfaction sexuelle (Steer \& Tiggemann, 2008). En outre, lorsqu'elle est associée à une impossibilité d'atteindre les standards imposés par les médias, cette autosurveillance contraint également les femmes à une comparaison ascendante (c'est-àdire à une comparaison avec un individu que l'on estime supérieur à soi), qui est potentiellement délétère pour leur estime d'elles-mêmes (Liss \& 
Erchull, 2015). Ces conséquences entraînent à leur tour des comportements visant à résorber cet écart entre le soi et l'idéal de beauté (p. ex. des troubles alimentaires : Moradi, Dirks, \& Matteson, 2005), une tendance à fumer davantage pour perdre du poids (Fiissel \& Lafreniere, 2006), ou une attitude et des comportements favorables vis-à-vis de la chirurgie esthétique (Forbes, Jobe, \& Revak, 2006 ; Henderson-King \& HendersonKing, 2005). D'autres comportements ou attitudes sont liés à l'impossibilité d'atteindre et de maintenir ces standards (p. ex. les symptômes dépressifs : Miner-Rubino, Twenge, \& Fredrickson, 2002; Muehlenkamp \& Saris-Baglama, 2002 ; Tolman, Impett, Tracy, \& Michael, 2006).

La Figure 1 propose un modèle révisé de la théorie de l'objectification (Fredrickson \& Roberts, 1997) plus complet, enrichi et actuel des avancées majeures dans ce domaine. En effet, ce modèle théorique distingue les concepts d'auto-objectification et d'autosurveillance souvent confondus dans la littérature. De plus, il énonce plus précisément les conséquences délétères les plus fréquentes sur la santé psychologique et mentale. Enfin, il met l'accent sur les facteurs modérateurs, prédicteurs et antécédents du concept d'auto-objectification, qui font l'objet de discussions étayées scientifiquement plus loin dans la présente revue de la littérature.

En somme, les études dans ce domaine sont concluantes et s'accordent pour constater un impact pernicieux de ce phénomène sur la santé mentale, l'auto-perception, la cognition, le bien-être ou encore la santé physique. Une question peut dès lors être posée : sommes-nous tous et toutes concernés par ce phénomène?

\section{MODÉRATEURS}

Certains facteurs sont responsables de variations concernant la sensibilité à l'auto-objectification. Dans cette section, nous relevons la plupart des éléments modérateurs de l'auto-objectification tels que le genre, la culture, l'orientation sexuelle, l'âge ou encore la compassion pour soimême.

\section{Genre}

Depuis l'émergence des mouvements féministes en Occident, le concept d'objectification a principalement été appliqué aux femmes 
Pixellence - 07-07-20 11:48:27 - (c) Humensis

RE0108 U000 - Oasys 19.00x - Page 329 - E1

Revue Annee psychologique 120-3 - Dynamic layout $0 \times 0$

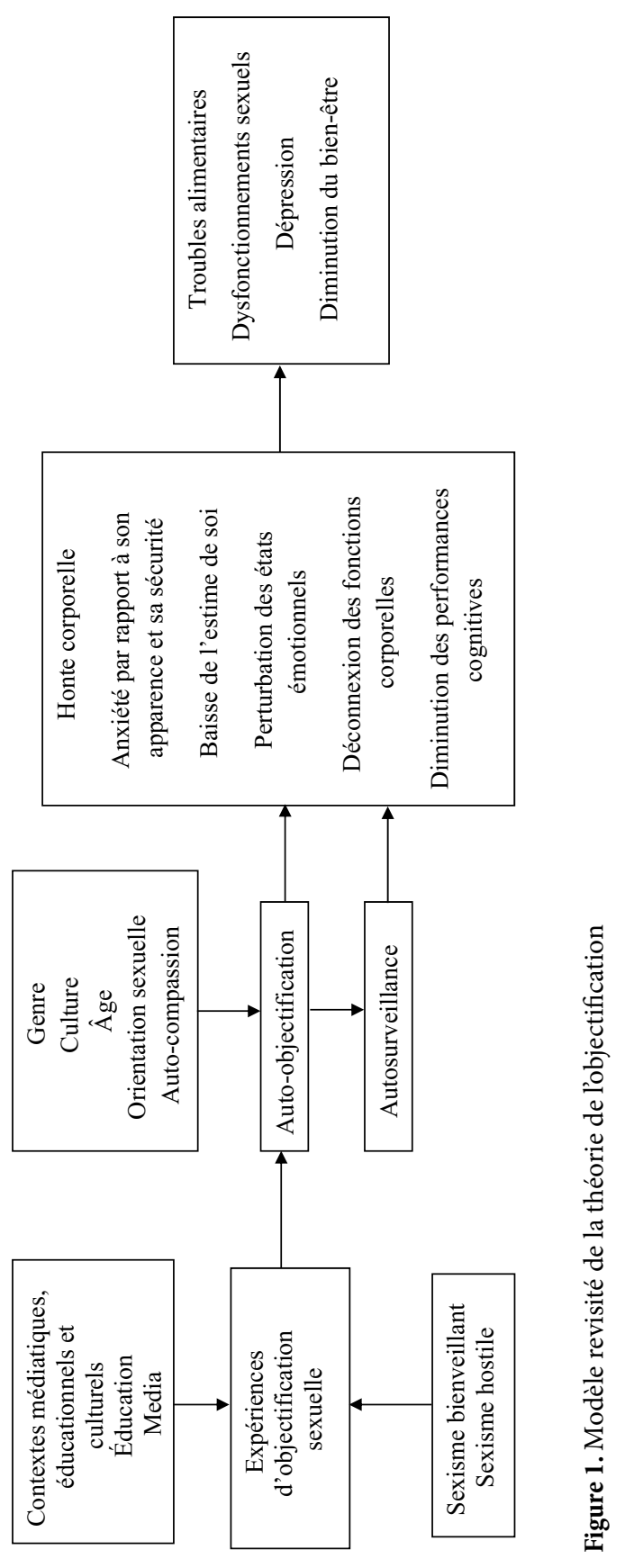


Pixellence - 07-07-20 11:48:27 - (c) Humensis

RE0108 U000 - Oasys 19.00x - Page 330 - E1

Revue Annee psychologique 120-3 - Dynamic layout $0 \times 0$

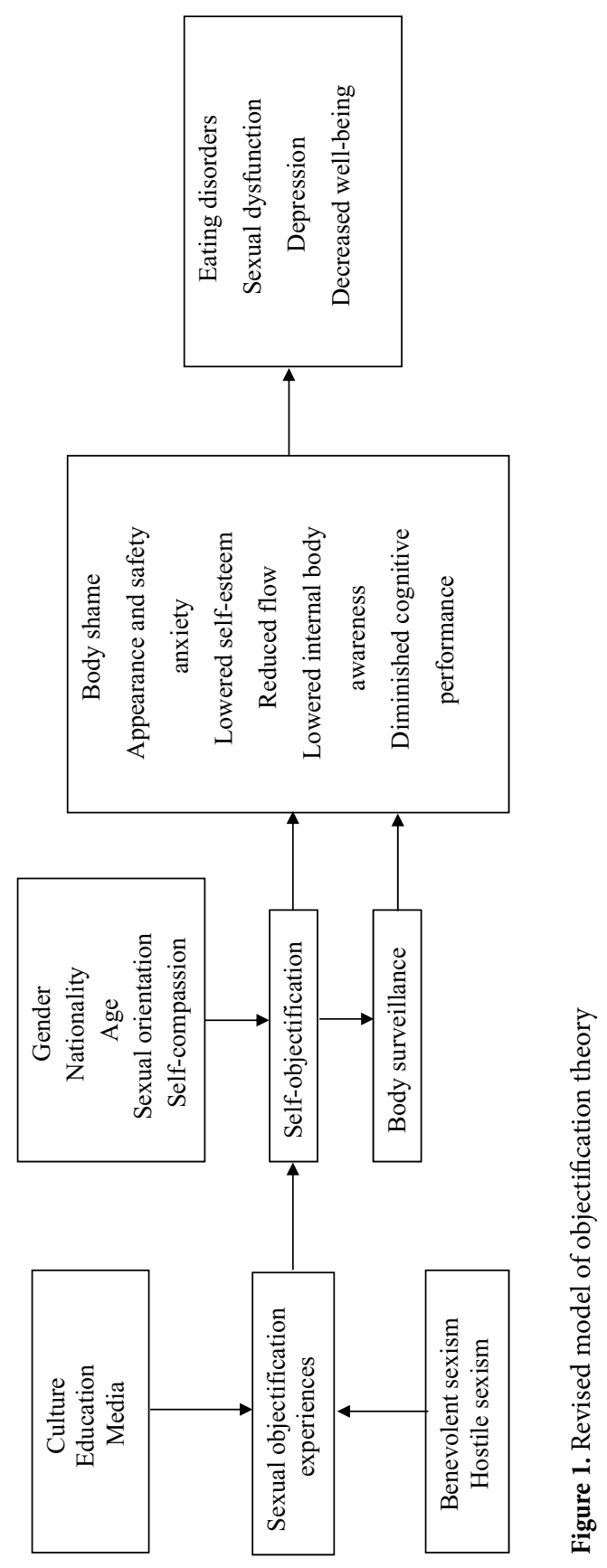


(Huebner \& Fredrickson, 1999; McKinley, 2006 ; Wolf, 1991). Récemment, des travaux ont mis en évidence que ce phénomène concerne également les hommes (p. ex., Daniel \& Bridges, 2010 ; Daniel, Bridges, \& Martens, 2014 ; Morrison, Morrison, \& Hopkins, 2003 ; Pope, Olivardia, Gruber, \& Borowiecki, 1999), même si ce sont d'autres attributs corporels qui sont impliqués en fonction du genre (p. ex., idéal de musculation, Leit, Pope, \& Gray, 2001 ; McCreary \& Sasse, 2000). En effet, la littérature suggère que les médias génèrent un environnement sexuellement objectifiant qui encourage les hommes à être musclés et les femmes à maigrir (Johnson, McCreary, \& Mills, 2007). Par conséquent, ces images objectifiantes renforcent les rôles de genre traditionnels, comme celui de l'homme dominant, viril et actif face à une femme soumise, féminine et passive (p. ex., Calogero \& Jost, 2011 ; Dardenne, Delacolette, Grégoire, \& Lecocq, 2006; Glick \& Fiske, 1996, 2001 ; Shepherd et al., 2010 ; Sibley \& Wilson, 2004). En effet, une étude menée aux États-Unis a mis en évidence que le pourcentage d'hommes rapportant un sentiment d'insatisfaction corporelle est passé de $15 \%$ en 1972 à $43 \%$ en 1985 (Gardner, 1997). Par exemple, l'exposition à des images stéréotypées et idéalisées d'hommes (p. ex., musculature affinée) génère chez ces derniers de l'insatisfaction corporelle (p. ex., Harrison \& Cantor, 1997 ; Lavine, Sweeney, \& Wagner, 1999), un sentiment d'être trop petits ou sous-développés (Luciano, 2001), des troubles alimentaires (Morry \& Staska, 2001), une diminution de l'estime de soi et une volonté de faire davantage d'exercices physiques (Murnen, Smolak, Mills, \& Good, 2003 ; Strelan \& Hargreaves, 2005), tout cela résultant principalement d'une plus grande insatisfaction face à leur aspect physique (p. ex., Arbour \& Ginis, 2006; Hatoum \& Belle, 2004).

\section{Culture}

Remarquons que la plupart des débats, exemples et observations empiriques à partir desquels se construit la théorie de l'objectification concernent des échantillons de femmes blanches issues de sociétés occidentales. En effet, la grande majorité des recherches ont été menées aux États-Unis et en Europe de l'Ouest. Ultérieurement, d'autres équipes de chercheurs ont étudié ce phénomène dans des contextes différents et ont mis en évidence que l'auto-objectification engendrait également des conséquences négatives chez des Afro-Américains, des Hispaniques et des Américains d'origine asiatique vivant aux États-Unis (Hebl, King, \& Lin, 
2004). De plus, certaines études tendent à démontrer que les conséquences négatives de l'auto-objectification apparaissent également dans différents pays comme le Népal (Crawford et al., 2008), l'Inde (Loughnan et al., 2015), la Thailande (Wollast, Coertjens, Bernard, \& Klein, 2019), la Russie (Wollast, Riemer, Gervais, Grigoryan, Bernard \& Klein, 2019), la république des Fidji (Becker, 2004), la Corée du Sud (Lyu, 2016) ou encore la Chine (Teng et al., 2016; Teng et al., 2017). En somme, ces études suggèrent que le processus d'auto-objectification prédit négativement des indicateurs de santé mentale et physique dans de nombreux pays dont les caractéristiques culturelles sont diverses et variées.

Par ailleurs, en plus d'être influencés culturellement, les normes et codes esthétiques de beauté évoluent avec le temps. Par exemple, dans les sociétés antiques grecques et romaines, ou sous l'ancien régime, la noblesse se devait d'avoir le teint pâle et blanchâtre reflétant un idéal de pureté, de virginité, une âme propre immaculée démontrant une humanité sans tache et sans faille (Vigarello, 2004). Cette extrême blancheur était considérée comme un marqueur social, réservée à une élite oisive et riche, si on les compare aux visages mats et bronzés du peuple, de la classe laborieuse souvent considérée comme sale et impure. Aujourd'hui, certains pays d'Afrique, d'Asie ou encore l'Inde vouent un véritable culte à la peau blanche (Li et al., 2008). Selon Chaipraditkul (2013), dans la plupart des cultures asiatiques, la beauté est extrêmement importante pour les femmes car elle peut conduire à de nombreux avantages sociaux à condition de respecter les critères esthétiques (teint de peau pâle, ouverture des yeux, forme du nez...). Il est donc peu surprenant de constater que le niveau d'auto-objectification sexuelle est très élevé dans ces pays (Lyu, 2016; Teng et al., 2016; Teng et al., 2017). Dans ce contexte, Von Feigenblatt (2010) introduit les termes de culture subjective (locale et propre à la culture d'origine) et de culture objective (idéaux imposés par une culture dominante) pour décrire l'influence des systèmes de pensées occidentaux et indiens sur les normes de beauté dans l'Asie du Sud-Est. En résumé, ceci illustre bien le caractère évolutif et adaptatif du phénomène d'objectification sexuelle en fonctions des contextes culturels et temporels et de leurs interactions.

\section{Auto-compassion}

Comme nous l'avons vu à travers cette revue de la littérature, l'autoobjectification semble générer une série de conséquences délétères sur la 
santé mentale et physique. Actuellement, très peu d'études se sont penchées sur les processus psychologiques susceptibles d'apaiser ces conséquences négatives. À ce propos, plusieurs travaux suggèrent que la capacité à être bienveillant et compréhensif envers soi-même (l'auto-compassion; Neff, 2003b) pourrait constituer une stratégie de résilience efficace.

L'auto-compassion - ou la compassion pour soi-même - est caractérisée par une attitude positive, bienveillante et compréhensive envers soimême (Neff, 2003a). Dans ce domaine, la littérature récente soutient l'idée qu'en désactivant le système de menace généré par l'objectification et en favorisant une restructuration cognitive positive, l'auto-compassion améliorerait la résilience émotionnelle («capacité à faire face à une situation difficile ou génératrice de stress » : Kline \& Short, 1991). En ce sens, les recherches suggèrent que les individus présentant un niveau élevé d'auto-compassion s'engageraient moins dans des processus d'autosurveillance, ressentiraient moins de honte corporelle et de dépression comparés aux individus qui manifestent un faible niveau d'auto-compassion (Liss \& Erchull, 2015). En effet, Wollast, Riemer, Bernard, Leys, Kotsou et Klein (2019) ont démontré qu'un faible niveau de compassion pour soi-même influençait négativement l'effet de la surveillance corporelle sur la dépression et le bonheur chez les femmes. Plus spécifiquement, chez les femmes qui obtiennent des scores faibles d'auto-compassion, la surveillance corporelle détériore la perception subjective du bonheur, et s'explique par un niveau de dépression plus élevé (Wollast, Riemer, Bernard, et al., 2019). De plus, certains auteurs ont constaté que l'autocompassion modère l'intensité du lien entre l'image du corps et l'estime de soi chez les adolescentes (Pisitsungkagarn, Taephant, \& Attasaranya, 2014). Spécifiquement, la relation entre l'image du corps et l'estime de soi devient moins marquée chez les adolescentes qui ont un niveau élevé d'auto-compassion. Dans ce même contexte, des résultats similaires concernant l'effet bénéfique de l'auto-compassion sur l'image du corps ont été démontrés chez les hommes (Wollast, Riemer, Sarda, \& Klein, 2019).

En outre, l'auto-compassion génère des effets bénéfiques sur l'image corporelle, y compris dans les milieux cliniques. Par exemple, les patients souffrant de troubles alimentaires qui ont un niveau élevé d'auto-compassion en début de traitement témoignent d'une diminution plus rapide des conséquences négatives liées à leur alimentation (Kelly, Carter, \& Borairi, 2013). De plus, l'auto-compassion semble jouer un rôle clef dans la relation entre l'image du corps et la détresse des femmes qui ont survécu à un cancer (Przezdziecki et al., 2012). Plus précisément, les chercheurs ont 
montré qu'un faible niveau d'auto-compassion est associé à un niveau élevé d'anxiété ainsi qu'à une image de soi négative chez les femmes ayant suivi un traitement complet contre le cancer (chirurgie, chimiothérapie, radiations). Récemment, certains travaux suggèrent que l'écoute d'enregistrements de séances de méditation de pleine conscience guidée et la pratique du yoga génèrent, par le biais d'une augmentation de l'autocompassion, un impact positif sur la manière dont les femmes perçoivent leur propre corps (Albertson, Neff, \& Dill-Shackleford, 2014; Cox, Ullrich-French, Cole, \& D'Hondt-Taylor, 2016; Cox, Ullrich-French, Howe, \& Cole, 2017). En somme, la compassion pour soi-même semble démontrer son importance dans le mécanisme de la résilience face à l'objectification sexuelle dans notre société contaminée par l'omniprésence des représentations féminines et masculines objectifiantes, trompeuses et non représentatives de sa population (pour une revue détaillée, voir Wollast, 2018).

De manière importante, les recherches considèrent souvent l'autocompassion comme un remède miracle contre toute une série de conséquences négatives sur l'image corporelle et la santé mentale. Pourtant, l'effet de cette variable est limité, car il n'opère qu'au niveau individuel. En effet, certaines contraintes collectives et sociales peuvent considérablement diminuer son impact. Par exemple, il serait bien insuffisant de renforcer le niveau d'auto-compassion d'une jeune fille pour qu'elle puisse plus facilement faire face aux regards concupiscents ou aux réflexions objectifiantes liés au harcèlement de rue. En d'autres mots, l'auto-compassion est une stratégie très personnelle qui, finalement, ne contribue pas à remettre en cause les inégalités entre les hommes et les femmes ainsi que les autres formes de discriminations (e.g., omniprésence des corps idéalisés, stéréotypés et sexualisés dans les médias). Il n'en reste pas moins que les travaux dans ce domaine mènent à envisager que l'auto-compassion ne soit pas uniquement un trait de personnalité individuelle mais puisse devenir, à certains égards, une norme sociale (e.g., culture de l'acceptation). En effet, on sait que dans d'autres domaines, des initiatives visant à transformer les normes sociales, concernant l'image du corps notamment, se sont avérées efficaces (Yager \& O’Dea, 2008).

\section{Caractéristiques socio-démographiques}

Différentes études mettent en évidence l'influence de l'âge et de l'orientation sexuelle sur l'auto-objectification (cf. Calogero, 2012). En 
effet, certaines études suggèrent que l'auto-objectification, la surveillance du corps, l'anxiété liée à l'apparence physique et les troubles alimentaires diminuent avec l'âge (Tiggemann \& Lynch, 2001). On constate également que l'exposition à des images objectifiantes génère de l'autosurveillance ainsi que de la honte corporelle chez les adolescents (Tiggemann \& Pickering, 1996) ainsi que chez les jeunes filles de 5 à 8 ans (Dohnt \& Tiggemann, 2006). D'autres chercheurs ont mis en évidence que l'autoobjectification était également ressentie différemment en fonction de l'orientation sexuelle (Martins, Tiggemann, \& Kirkbride, 2007 ; Tiggemann, Martins, \& Kirkbride, 2007). Par exemple, Kozak, Frankenhauser et Roberts (2009) ont observé que des hommes homosexuels s'auto-objectifient et objectifient davantage autrui que des hommes hétérosexuels. Dans la même veine, les recherches ont montré que les femmes lesbiennes étaient également touchées par les phénomènes d'auto-objectification, d'autosurveillance, de honte corporelle, d'internalisation de l'idéal de minceur ou encore des troubles alimentaires (Hill \& Fischer, 2008 ; Kozee et al., 2007 ; Kozee \& Tylka, 2006 ; Moradi, Dirks, \& Matteson, 2005).

\section{LIMITES}

Cette section reprend les critiques majeures émises à l'encontre de la théorie actuelle et concerne principalement la représentativité des échantillonnages, le manque de comparaisons interculturelles ainsi que les problèmes liés à l'opérationnalisation des mesures.

\section{Représentativité des échantillonnages}

La grande majorité des études empiriques dans le domaine de l'objectification se sont focalisées sur des populations de femmes hétérosexuelles blanches occidentales, souvent étudiantes en psychologie. En 2008, Moradi et Huang constataient que $75 \%$ des études dans ce domaine ont été menées sur des femmes américaines, ce qui représente seulement $2.2 \%$ de la population mondiale. Suite à ces observations, nous constatons une expansion de la littérature dans les groupes spécifiques, basés sur l'orientation sexuelle, la culture, l'âge, la langue, la religion, le statut socio-économique et l'état civil, la ville de résidence, l'origine des parents et le genre. Cependant, le pourcentage de recherches effectuées dans des 
contextes particuliers reste très faible. Par conséquent, si l'on veut dégager une vision représentative et approfondie des mécanismes liés à l'objectification, l'étude de ce phénomène dans des populations sous-représentées au regard du constat de Moradi et Huang reste minoritaire.

À cet égard, rares sont les études effectuant des comparaisons interculturelles (p. ex., Loughnan et al., 2015; Wollast, Coertjens, Bernard, \& Klein, 2019). Or, identifier les facteurs qui expliquent ces différences culturelles permettrait de renforcer la théorie de l'objectification en permettant d'identifier les mécanismes intervenant dans le phénomène d'objectification et ses conséquences sur le bien-être.

\section{Opérationnalisation et mesures}

En psychologie sociale, l'approche quantitative via le questionnaire d'auto-objectification (Self-objectification Questionnaire, Fredrickson et al., 1998) est la plus communément utilisée pour étudier le phénomène d'auto-objectification. Plus spécifiquement, les participants y sont invités à classer par ordre d'importance dix attributs corporels dans la façon dont ils perçoivent leur apparence physique. La première moitié des attributs sont observables (poids, avoir des muscles sculptés/fermes, mensurations [poitrine, taille...], sex-appeal et charme) et la seconde moitié correspond à des attributs non observables (coordination physique [agilité, souplesse], santé, force, niveau d'endurance, condition physique). Plus le participant accorde d'importance aux attributs visibles, plus son score d'auto-objectification est élevé.

Bien que très répandue dans ce domaine, cette mesure n'est pas sans limites. En effet, ce questionnaire a été construit en accord avec les idéaux de beauté occidentaux et oriente les items sur les standards de beauté de la société américaine (p. ex., idéal de minceur et de musculature). Or, il est bien établi que d'autres attributs peuvent également jouer un rôle dans la manière dont les hommes et les femmes perçoivent leur propre corps. C'est le cas notamment de la couleur de peau (voir Buchanan, Fischer, Tokar, \& Yoder, 2008 pour une revue), mais aussi des rides, des cheveux blancs, des cicatrices, etc.

En outre, les mesures les plus fréquemment utilisées pour étudier le phénomène d'auto-objectification ne sont pas sans limites. En effet, il n'est pas rare de trouver des biais de genre dans les échelles mesurant les conséquences délétères. Par exemple, trois items sur huit pour l'échelle de honte corporelle (McKinley \& Hyde, 1996) se focalisent sur le poids 
alors qu'aucun item ne pose la question de la taille des muscles. Par conséquent, ceci pourrait expliquer pourquoi les femmes rapportent souvent des scores de honte corporelle plus élevés que les hommes ( $c f$. Idéal de minceur $v s$. Idéal de musculature). Par ailleurs, les chercheuses considèrent d'un point de vue théorique que la surveillance corporelle conduit à la honte corporelle. Or, une position alternative pourrait être envisagée à savoir que la honte corporelle pousse à la surveillance du corps. Ceci questionne la validité du modèle théorique proposé sur base de la causalité des variables utilisées. Enfin, bien que l'étude des différences culturelles soit en pleine expansion, l'interprétation du sens des échelles peut varier d'une culture à l'autre. Par exemple, l'autosurveillance pourrait ne pas refléter le type de surveillance corporelle qui déclenche le sentiment de honte dans les cultures orientales. En effet, cette échelle est fortement basée sur des comparaisons interpersonnelles, alors que, dans les cultures orientales, la honte corporelle ne découle pas nécessairement de comparaisons avec d'autres, mais de l'image que l'on projette sur le public. Par exemple, si le manque d'hygiène génère de la honte dans certaines cultures orientales parce que l'on ne respecte pas la personne avec laquelle nous communiquons, une mauvaise hygiène est considérée comme un signe de manque de respect de soi dans la culture occidentale. En d'autres termes, le sentiment de honte corporelle résultant de l'autosurveillance peut varier d'une culture à l'autre (e.g. cultures individualistes ou collectivistes). En conséquence, la mesure d'autosurveillance et de la honte corporelle utilisée dans la littérature actuelle pourrait ne pas refléter exactement la même signification d'une culture à l'autre.

\section{RECHERCHES COMPLÉMENTAIRES DE LA THÉORIE DE L'OBJECTIFICATION}

\section{Auto-objectification instrumentale}

La liberté que possède chaque femme de disposer librement de son propre corps fait l'objet de débats au sein du mouvement féministe. La théorie de l'objectification formulée par Fredrickson et Roberts (1997) a récemment été remise en question en raison du rôle de victime passive qui est attribué à la femme dans le processus d'internalisation de l'objectification (c'est-à-dire d'auto-objectification) et de reproduction du système 
patriarcal dans lequel elle s'insère malgré elle (Erchull, Liss, \& Lichiello, 2013; Erchull \& Liss, 2014). En effet, plusieurs travaux suggèrent que certaines femmes ressentent un sentiment de contrôle et d'auto-détermination en choisissant activement d'instrumentaliser leur apparence physique à leur avantage (Calogero, 2013; Erchull, Liss, \& Lichiello, 2013; Liss, Erchull \& Ramsey, 2010). Ces objectifs étant d'ordre individuel (p. ex., approbation sociale, bénéfices matériels, pouvoir interpersonnel, sentiment d'agentivité ; Anderson, 2015) ou collectif (p. ex., Femen ; Klein, Allen, Bernard, \& Gervais, 2014).

L'émergence de ce phénomène s'expliquerait par l'évolution des stéréotypes féminins allant d'une représentation passive et soumise de la femme (p. ex., la ménagère des années cinquante) vers celle d'une femme à la fois sexy, sûre d'elle, indépendante et disposant d'une certaine forme de pouvoir (Gill, 2008 ; Twenge, 2001). Ces constats ont donné lieu à une nouvelle ligne de recherche fondée sur la théorie de l'objectification (De Wilde, Casini, Wollast, \& Demoulin, 2019). En s'appuyant sur la définition de Nussbaum (1995), Calogero (2013) avance que, s'il y a plusieurs manières d'objectifier quelqu'un, il devrait également y avoir plusieurs manières de s'auto-objectifier. Cette auteure suggère notamment d'examiner la possibilité que les femmes instrumentalisent leur propre corps de manière active et intentionnelle (Calogero, 2013). À l'heure actuelle, le caractère illusoire, ou non, et l'aspect individuel ou collectif du pouvoir généré par l'assertivité sexuelle restent largement débattus (Anderson, 2015 ; Lamb \& Peterson, 2012). Par exemple, il est possible que les femmes qui instrumentalisent leur apparence de manière intentionnelle bénéficient de cette forme d'auto-détermination. En effet, les femmes plus assertives sexuellement éprouveraient moins de difficulté à exprimer leurs préférences lors de relations sexuelles (Noar, Morokoff, \& Redding, 2002). Toutefois, certains chercheurs suggèrent qu'une incidence délétère s'exerce à un niveau structurel et sociétal. En effet, Gill $(2008,2012)$ souligne l'ambiguïté du message qui est transmis à travers la diffusion dans les médias de l'image d'une femme sûre d'elle et déterminée grâce à son attractivité physique. D'une part, ce message semble promettre à la femme une augmentation de son indépendance, de son autonomie et de son pouvoir. D'autre part, le fait de correspondre aux attentes des hommes en matière d'apparence est paradoxalement la condition de l'obtention de ce pouvoir. Ainsi, par le biais des médias, les femmes apprendraient à endosser activement, voire stratégiquement le statut d'objet sexuel (Allen \& Gervais, 2012). Pour aller plus loin, Gill (2008) propose qu'en instrumentalisant leur apparence, les femmes passent du statut de victime passive d'un système objectifiant à celui d'agent perpétuant activement 
l'idée selon laquelle le seul pouvoir des femmes réside dans leur apparence.

\section{Objectification d'autrui}

Comme nous l'avons illustré au travers des sections précédentes de cette revue de la littérature, la majorité des travaux réalisés dans le cadre de la théorie de l'objectification s'est penchée sur les conséquences de l'auto-objectification. En revanche, ces travaux sont peu informatifs concernant la perception que l'on peut avoir des individus objectifiés. Qu'entend-on par «objectification d'autrui » ou «objectification des autres " ? Comment les individus sexuellement objectifiés sont-ils perçus ?

Deux éléments sont centraux lorsque nous abordons le concept d'objectification d'autrui. Premièrement, la majorité des philosophes s'accordent à dire que considérer une personne comme un objet implique une forme de déshumanisation (Kant, 1797, 1993; Nussbaum, 1995 ; Papadaki, 2007, 2010). En d'autres termes, une personne objectifiée serait perçue comme possédant moins d'humanité. De nombreuses recherches ont mis en évidence que ce lien entre objectification et déshumanisation est bel et bien présent (e.g., Heflick et al., 2011 ; Heflick \& Goldenberg,2009 ; Vaes, Paladino, \& Puvia, 2011 ; Wollast, Puvia, Bernard, Tevichapong, \& Klein 2018 ; pour une revue de la littérature, voir Gervais, Bernard, Klein, \& Allen, 2013 ; Heflick \& Goldenberg, 2014). Deuxièmement, le processus d'objectification correspond à une perception fragmentée ou morcelée de la cible objectifiée. La philosophe Bartky (1990) suggère qu'une personne est objectifiée sexuellement lorsque cette dernière est abordée comme un corps, voire comme des parties de corps destinées à satisfaire les désirs sexuels de la personne qui l'objectifie (c'està-dire l'objectifieur). À ce titre, des recherches récentes ont mis en évidence que la sexualisation du corps induit une «objectification cognitive ": les corps sexualisés, à l'instar des objets, sont perçus de façon moins globale (Bernard, Rizzo et al., 2018; Bernard, Hanoteau et al., 2018) et plus fragmentée (Bernard, Content, Deltenre, \& Colin, 2018) que les corps non-sexualisés à un niveau précoce du traitement visuel (pour une revue de la littérature, voir Bernard, Gervais \& Klein, 2018). 


\section{CONCLUSION}

Le présent article constitue la première revue de la littérature sur la théorie de l'objectification en langue française. Élaborée à partir des études phares issues de plus de vingt années de recherches en psychologie, cette revue narrative offre aux intéressés une approche synthétique, critique et actuelle des enjeux sociétaux liés au phénomène d'objectification.

Ancrée insidieusement dans nos sociétés, l'objectification du corps est présente au quotidien dans les médias ainsi que dans les interactions sociales. En ce sens, les images médiatiques souvent sexualisées, stéréotypées ou idéalisées, associées aux rôles de genre traditionnels, conditionnent les individus à percevoir leur corps comme un simple objet. Cette auto-objectification est la source d'une série de conséquences néfastes sur la santé mentale et psychologique des individus. Fort heureusement, certains facteurs permettent de se prémunir des conséquences négatives générées par le contexte toxique de l'objectification. En effet, l'auto-compassion semble démontrer son importance dans le mécanisme de la résilience face à l'objectification sexuelle, favorisant une meilleure acceptation de son corps afin de moins ressentir les pressions délétères exercées par la société actuelle.

Enfin, bien que la théorie de l'objectification ait connu une expansion considérable, de nombreuses pistes restent à explorer en vue de renforcer nos connaissances théoriques dans ce domaine, mais également de contribuer à une meilleure qualité de vie chez les personnes concernées par ce phénomène.

\section{RÉFÉRENCES}

Albertson, E. R., Neff, K. D., \& Dill-Shackleford, K. E. (2014). Self-compassion and body dissatisfaction in women: a randomized controlled trial of a brief meditation intervention. Mindfulness, 6(3), 444-454. https://doi.org/10.1007/s12671-014-0277-3

Anderson, K. J. (2015). Modern misogyny: Anti-feminism in a post-feminist era. New York: Oxford University Press.

\author{
Arbour, K. P., \& Martin Ginis, K. A. \\ (2006). Effects of exposure to muscular \\ and hypermuscular media images on \\ young men's muscularity dissatisfaction \\ and body dissatisfaction. Body Image, 3, \\ 153-161. https://doi.org/10.1016/j.bodyim. \\ 2006.03.004 \\ Aubrey, J. S. (2006). Effects of sexually \\ objectifying media on self-objectification \\ and body surveillance in undergraduates:
}


Results of a 2-year panel study. Journal of Communication, 56(2), 366-386. https:// doi.org/10.1111/j.1460-2466.2006.00024.x

Bartky, S. L. (1990). Femininity and domination: Studies in the phenomenology of oppression. New York: Routledge.

Bauer, N. (2010). Beauvoir on the allure of self-objectification. Feminist Metaphysics, 117-129. https://doi.org/10.1007/978-90481-3783-1_8

Beauvoir, S. de (1949). Le deuxième sexe (t. II). Paris : Éditions Gallimard.

Becker, A. E. (2004). Television, disordered eating, and young women in Fiji: Negotiating body image and identity during rapid social change. Culture, Medicine and Psychiatry, 28(4), 533-559. https://doi.org/ 10.1007/s11013-004-1067-5

Berger, J. (1972). Ways of seeing. London, England: Penguin.

Bernard, P., Content, J., Deltenre, P., \& Colin, C. (2018). When the body becomes no more than the sum of its parts: The neural correlates of scrambled vs. intact sexualized bodies. NeuroReport, 29, 48-53. https://doi.org/10.1097/WNR.00000000000 00926

Bernard, P., Gervais, S., \& Klein, O. (2018). Objectifying objectification: When and why people are cognitively reduced to their parts akin to objects. European Review of Social Psychology, 29, 82-121. https:// doi.org/10.1080/10463283.2018.1471949

Bernard, P., Hanoteau, F., Gervais, S., Servais, L., Bertolone, I., Deltenre, P. \& Colin, C. (2019). Revealing clothing does not make the object: ERP evidences that cognitive objectification is driven by posture suggestiveness, not by revealing clothing. Personality and Social Psychology Bulletin, 45, 16-36. https://doi.org/10.1177/ 0146167218775690

Bernard, P., Rizzo, T., Hoonhorst, I., Deliens, G., Gervais, S., et al. (2018). The neural correlates of cognitive objectification: an ERP study on the body-inversion effect associated with sexualized bodies. Social and Psychological Personality Science, 9, 550-559. https://doi.org/ $10.1177 / 1948550617714582$

Bordo, S. (1993). Unbearable weight: Feminism, western culture and the body. Berkeley, CA: University of California Press.

Bordo, S. (1999). The male body: A new look at men in public and in private. New York: Farrar, Straus, and Giroux.

Buchanan, T. S., Fischer, A. R., Tokar, D. M., \& Yoder, J. D. (2008). Testing a culture-specific extension of objectification theory regarding African American women's body image. The Counseling Psychologist, 36, 697-718. https://doi.org/ $10.1177 / 0011000008316322$

Calogero, R. M. (2012). Objectification theory, self-objectification, and body image. Encyclopedia of Body Image and Human Appearance, 574-580. https://doi.org/10.10 16/b978-0-12-384925-0.00091-2

Calogero, R. M. (2013). On objects and actions: Situating self-objectification in a system justification context. Objectification and (de)humanization, 97-126. https:// doi.org/10.1007/978-1-4614-6959-9_5

Calogero, R. M., \& Jost, J. T. (2011). Selfsubjugation among women: exposure to sexist ideology, self-objectification, and the protective function of the need to avoid closure. Journal of Personality and Social Psychology, 100(2), 211-228. https:// doi.org/10.1037/a0021864

Connell, N., \& Wilson, C. (1974). Rape: the first sourcebook for women. Plume.

Cox, A. E., Ullrich-French, S., Cole, A. N., \& D'Hondt-Taylor, M. (2016). The role of state mindfulness during yoga in predicting self-objectification and reasons for exercise. Psychology of Sport and Exercise, 22, 321-327. https://doi.org/10.1016/ j.psychsport.2015.10.001

Cox, A. E., Ullrich-French, S., Howe, H. S., \& Cole, A. N. (2017). A pilot yoga physical education curriculum to promote 
positive body image. Body Image, 23, 1-8. https://doi.org/10.1016/j.bodyim.2017.07.007

Crawford, M., Lee, I.-C., Portnoy, G., Gurung, A., Khati, D., Jha, P., \& Regmi, A. C. (2008). Objectified body consciousness in a developing country: A comparison of mothers and daughters in the US and Nepal. Sex Roles, 60(3-4), 174-185. https://doi.org/ 10.1007/s11199-008-9521-4

Daniel, S., \& Bridges, S. K. (2010). The drive for muscularity in men: Media influences and objectification theory. Body Image, 7(1), 32-38. https://doi.org/10.1016/ j.bodyim.2009.08.003

Daniel, S., Bridges, S. K., \& Martens, M. P. (2014). The development and validation of the Male Assessment of Self-Objectification (MASO). Psychology of Men \& Masculinity, 15(1), 78-89. https://doi.org/10.1037/a003 1518

Dardenne, B., Delacollette, N., Grégoire, C., \& Lecocq, D. (2006). Structure latente et validation de la version française de l'Ambivalent Sexism Inventory : l'échelle de sexisme ambivalent. L'Année Psychologique, 106(02), 235-263. https://doi.org/ $10.4074 / \mathrm{s} 0003503306002041$

De Wilde, M., Casini, A., Wollast, R., \& Demoulin, S. (soumis). Sex is power belief and women's mental health: Analysis of the mediating role of self-objectification and sexual subjectivity on eating disorders and sexual satisfaction. Manuscrit en révision.

Dohnt, H., \& Tiggemann, M. (2006). The contribution of peer and media influences to the development of body satisfaction and self-esteem in young girls: A prospective study. Developmental Psychology, 42, 929-936. https://doi.org/10.1037/0012-16 49.42.5.929

Dworkin, A. (1985). Against the male flood: Censorship, pornography, and equality. Harvard Women's Law Journal, 8, 1-29

Erchull, M. J., \& Liss, M. (2014). The object of one's desire: How perceived sexual empowerment through objectification is related to sexual outcomes. Sexuality \& Culture, 18(4), 773-788. https:// doi.org/10.1007/s12119-013-9216-Z

Erchull, M. J., Liss, M., \& Lichiello, S. (2013). Extending the negative consequences of media internalization and selfobjectification to dissociation and selfharm. Sex Roles, 69(11-12), 583-593. https://doi.org/10.1007/s11199-013-0326-8

Fiissel, D. L., \& Lafreniere, K. D. (2006). Weight control motives for cigarette smoking: Further consequences of the sexual objectification of women? Feminism \& Psychology, 16(3), 327-344. https:// doi.org/10.1177/0959353506067850

Forbes, G. B., Jobe, R. L., \& Revak, J. A. (2006). Relationships between dissatisfaction with specific body characteristics and the Sociocultural Attitudes Toward Appearance Questionnaire-3 and Objectified Body Consciousness Scale. Body Image, 3(3), 295-300. https://doi.org/10.1016/ j.bodyim.2006.07.003

Fredrickson, B. L., \& Roberts, T. A. (1997). Objectification theory: Toward understanding women's lived experiences and mental health risks. Psychology of Women Quarterly, 21, 173-206. https://doi.org/ 10.1111/j.1471-6402.1997.tb00108.x

Fredrickson, B. L., Roberts, T. A., Noll, S. M., Quinn, D. M., \& Twenge, J. M. (1998). That swimsuit becomes you: Sex differences in self-objectification, restrained eating, and math performance. Journal of Personality and Social Psychology, 75(5), 269-284. https://doi.org/10.1037/h0090332

Garner, D. M. (1997). The 1997 body image survey results. Psychology Today, 30, 30-45.

Gay, R. K., \& Castano, E. (2010). My body or my mind: The impact of state and trait objectification on women's cognitive resources. European Journal of Social Psychology, 40(5), 695-703. https://doi.org/ 10.1002/ejsp.731 
Gervais S. J., Bernard P., Klein O., Allen J. (2013) Toward a unified theory of objectification and dehumanization. In S. J. Gervais (Ed.). 60th Nebraska Symposium on Motivation: Objectification and (de)humanization (pp. 1-23). New York, NY: Springer. https://doi.org/10.1007/978-1-46146959-9_1

Gill, R. (2008). Empowerment/sexism: Figuring female sexual agency in contemporary advertising. Feminism \& Psychology, 18(1), 35-60. https://doi.org/10.1177/ 0959353507084950

Gill, R. (2012). Media, empowerment and the 'sexualization of culture'debates. Sex Roles, 66(11-12), 736-745. https://doi.org/ 10.1007/s11199-011-0107-1

Glick, P., \& Fiske, S. T. (1996). The Ambivalent Sexism Inventory: Differentiating hostile and benevolent sexism. Journal of Personality and Social Psychology, 70, 491512. https://doi.org/10.1037/0022-3514.70. 3.491

Glick, P., \& Fiske, S. T. (2001). An ambivalent alliance: Hostile and benevolent sexism as complementary justifications for gender inequality. American Psychologist, 56, 109-118. https://doi.org/10.1037//0003066x.56.2.109

Green, L. (2000). Pornographies. Journal of Political Philosophy, 8(1), 27-52. https:// doi.org/10.1111/1467-9760.00091

Greenleaf, C. (2005). Self-objectification among physically active women. Sex Roles, 52(1-2), 51-62. https://doi.org/10.1007/ s11199-005-1193-8

Haslanger, S. (2012). On being objective and being objectified. Resisting Reality, 3582. https://doi.org/10.1093/acprof:oso/978 0199892631.003.0001

Harrison, K., \& Cantor, J. (1997). The relationship between media consumption and eating disorders. Journal of Communication, 47(1), 40-67. https://doi.org/10.1111/ j.1460-2466.1997.tb02692.x
Hatoum, I. J., \& Belle, D. (2004). Mags and abs: Media consumption and bodily concerns in men. Sex Roles, 51(7/8), 397407. https://doi.org/10.1023/b:sers.0000049 229.93256 .48

Hebl, M. R., King, E. B., \& Lin, J. (2004). The swimsuit becomes us all: Ethnicity, gender, and vulnerability to self-objectification. Personality and Social Psychology Bulletin, 30(10), 1322-1331. https:// doi.org/10.1177/0146167204264052

Heflick, N. A., \& Goldenberg, J. (2009). Objectifying Sarah Palin: Evidence that objectification causes women to be perceived as less competent and less fully human. Journal of Experimental Social Psychology, 45, 598-601. https://doi.org/10.1016/ j.jesp.2009.02.008

Heflick, N. A., Goldenberg, J. L., Cooper, D. P., \& Puvia, E. (2011). From women to objects: Appearance focus, target gender, and perceptions of warmth, morality and competence. Journal of Experimental Social Psychology, 47, 572-581. https://doi.org/ 10.1016/j.jesp.2010.12.020

Heflick, N. A., \& Goldenberg, J. L. (2014). Seeing eye to body. Current Directions in Psychological Science, 23(3), 225-229. https://doi.org/10.1177/0963721414531599

Henderson-King, D., \& Henderson-King, E. (2005). Acceptance of cosmetic surgery: Scale development and validation. Body Image, 2(2), 137-149. https://doi.org/ 10.1016/j.bodyim.2005.03.003

Hill, M. S., \& Fischer, A. R. (2008). Examining Objectification Theory. The Counseling Psychologist, 36(5), 745-776. https:// doi.org/10.1177/0011000007301669

Horney, K. (1967). Genesis of castration complex in women. In H. Kelman (Ed.). Feminine psychology (pp. 37-53). New York, NY: Norton.

Huebner, D. M., \& Fredrickson, B. L. (1999). Gender differences in memory 
perspectives: Evidence for self-objectification in women. Sex Roles, 41(5), 459-467. https://doi.org/10.1023/a:1018831001880

Johnson, P. J., McCreary, D. R., \& Mills, J. S. (2007). Effects of exposure to objectified male and female media images on men's psychological well-being. Psychology of Men \& Masculinity, 8, 95-102. https:// doi.org/10.1037/1524-9220.8.2.95

Kabat-Zinn, J. (1982). An outpatient program in behavioral medicine for chronic pain patients based on the practice of mindfulness meditation: Theoretical considerations and preliminary results. General Hospital Psychiatry, 4(1), 33-47. https://doi.org/10.1016,/0163-

8343(82)90026-3

Kahalon R., Shnabel, N., \& Becker, J. C. (2018) Experimental studies on state selfobjectification: A review and an Integrative Process Model. Frontiers in Psychology, 9. https://doi.org/10.3389/fpsyg.2018.01268

Kant, I. (1993). Métaphysique des mours. Paris : Vrin.

Kelly, A. C., Carter, J. C., \& Borairi, S. (2013). Are improvements in shame and self-compassion early in eating disorders treatment associated with better patient outcomes? International Journal of Eating Disorders, 47(1), 54-64. https://doi.org/ 10.1002/eat.22196

Klein, O., Allen, J., Bernard, P., \& Gervais, S. J. (2014). Angry naked ladies: How stereotyping and sexual objectification may be used to transform social systems. In D. Sindic, M. Barreto \& R. Costa-Lopes (Eds.). The intersection between power and identity (pp. 71-93). New York: Psychology Press.

Kline, B. E., \& Short, E. B. (1991). Changes in emotional resilience: Gifted adolescent boys. Roeper Review, 13(4), 184-187. https://doi.org/10.1080/ 02783199109553354
Kozak, M., Frankenhauser, H., \& Roberts, T.-A. (2009). Objects of desire: Objectification as a function of male sexual orientation. Psychology of Men \& Masculinity, 10(3), 225-230. https://doi.org/10.1037/ a0016257

Kozee, H. B., \& Tylka, T. L. (2006). A test of Objectification Theory with lesbian women. Psychology of Women Quarterly, 30(4), 348-357. https://doi.org/10.1111/ j.1471-6402.2006.00310.x

Kozee, H. B., Tylka, T. L., AugustusHorvath, C. L., \& Denchik, A. (2007). Development and psychometric evaluation of the Interpersonal Sexual Objectification Scale. Psychology of Women Quarterly, 31(2), 176-189. https://doi.org/10.1111/ j.1471-6402.2007.00351.x

Krawczyk, R., \& Thompson, J. K. (2015). The effects of advertisements that sexually objectify women on state body dissatisfaction and judgments of women: The moderating roles of gender and internalization. Body Image, 15, 109-119. https://doi.org/ 10.1016/j.bodyim.2015.08.001

LaCroix, J. M., \& Pratto, F. (2015). Instrumentality and the denial of personhood: The social psychology of objectifying others. Revue Internationale de Psychologie Sociale, 28(1), 183-211.

Lamb, S., \& Peterson, Z. D. (2012). Adolescent girls' sexual empowerment: Two feminists explore the concept. Sex Roles, 66, 703-712. https://doi.org/10.1007/ s11199-011-9995-3

Langton, R. (2009). Sexual solipsism: Philosophical essays on pornography and objectification. Oxford: Oxford University Press.

Lavine, H., Sweeney, D., \& Wagner, S. H. (1999). Depicting women as sex objects in Television advertising: Effects on body dissatisfaction. Personality and Social Psychology Bulletin, 25(8), 1049-1058. https:// doi.org/10.1177/01461672992511012 
Leit, R. A., Pope, H. G., \& Gray, J. J. (2001). Cultural expectations of muscularity in men: The evolution of Playgirl centerfolds. International Journal of Eating Disorders, 29(1), 90-93. https://doi.org/ 10.1002/1098-108x(200101)29:1<90::aideat $15>3.3 \cdot \operatorname{co} ; 2-6$

Li, E. P., Min, H. J., Belk, R. W., Kimura, J., \& Bahl, S. (2008). Skin lightening and beauty in four Asian cultures. Advances in Consumer Research, 35, 444-449.

Liss, M., Erchull, M. J., \& Ramsey, L. R. (2010). Empowering or oppressing? Development and exploration of the Enjoyment of Sexualization Scale. Personality and Social Psychology Bulletin, 37(1), 55-68. https://doi.org/10.1177/0146167210386119

Liss, M., \& Erchull, M. J. (2015). Not hating what you see: Self-compassion may protect against negative mental health variables connected to self-objectification in college women. Body Image, 14, 5-12. https://doi.org/10.1016/

j.bodyim.2015.02.006

Loughnan, S., Fernandez-Campos, S., Vaes, J., Anjum, G., Aziz, M., Harada, C., Holland, E., Singh, I, Puvia, E., \& Tsuchiya, K. (2015). Exploring the role of culture in sexual objectification: A seven nations study. Revue Internationale de Psychologie Sociale, 28(1), 125-152.

Loughnan, S., Haslam, N. Murnane, T., Vaes, J., Reynolds, C., \& Suitner, C. (2010). Objectification leads to depersonalization: The denial of mind and moral concern to objectified others. European Journal of Social Psychology, 40, 709-717. https:// doi.org/10.1002/ejsp.755

Luciano, L. (2001). Looking good: Male body image in modern America. New York: Hill and Wang.

Lyu, S. O. (2016). Travel selfies on social media as objectified self-presentation. Tourism Management, 54, 185-195. https:// doi.org/10.1016/j.tourman.2015.11.001
MacKinnon, C. A. (1987). Feminism unmodified: Discourses on life and law. Cambridge, MA: Harvard University Press. MacKinnon, C. A. (1989). Toward a feminist theory of the state. Cambridge, UK: Harvard University Press.

Martins, Y., Tiggemann, M., \& Kirkbride, A. (2007). Those speedos become them: The role of self-objectification in gay and heterosexual men's body image. Personality and Social Psychology Bulletin, 33(5), 634$647 . \quad$ https://doi.org/10.1177/ 0146167206297403

Marx, K. (2007). Les manuscrits économicophilosophiques de 1844, trad. Franck Fischbach. Paris : Vrin.

McCreary, D. R., \& Sasse, D. K. (2000). An exploration of the drive for muscularity in adolescent boys and girls. Journal of American College Health, 48(6), 297-304. https:// doi.org/10.1080/07448480009596271

McKinley, N. M., \& Hyde, J. S. (1996). The Objectified Body Consciousness Scale development and validation. Psychology of Women Quarterly, 20(2), 181-215. https:// doi.org/10.1111/j.1471-

6402.1996.tb00467.x

McKinley, N. M. (1999). Women and objectified body consciousness: Mothers' and daughters' body experience in cultural, developmental, and familial context. Developmental Psychology, 35, 760-769. https:// doi.org/10.1037/0012-1649.35.3.760

McKinley, N. M. (2006). The developmental and cultural contexts of objectified body consciousness: A longitudinal analysis of two cohorts of women. Developmental Psychology, 42(4), 679-687. https:// doi.org/10.1037/0012-1649.42.4.679

Miner-Rubino, K., Twenge, J. M., \& Fredrickson, B. L. (2002). Trait self-objectification in women: Affective and personality correlates. Journal of Research in Personality, 36(2), 147-172. https://doi.org/10.10 06/jrpe.2001.2343 
Pixellence - 07-07-20 11:48:28 - (c) Humensis

RE0108 U000 - Oasys 19.00x - Page 346 - E1

Revue Annee psychologique 120-3 - Dynamic layout $0 \times 0$

Moradi, B., Dirks, D., \& Matteson, A. V. (2005). Roles of sexual objectification experiences and internalization of standards of beauty in eating disorder symptomatology: A test and extension of Objectification Theory. Journal of Counseling Psychology, 52(3), 420-428. https:// doi.org/10.1037/0022-0167.52.3.420

Moradi, B., \& Huang, Y.-P. (2008). Objectification theory and psychology of women: A decade of advances and future directions. Psychology of Women Quarterly, 32, 377-398. https://doi.org/10.1111/j.1471 $-6402.2008 .00452 . x$

Morrison, T. G., Morrison, M. A., \& Hopkins, C. (2003). Striving for bodily perfection? An exploration of the drive for muscularity in Canadian men. Psychology of Men \& Masculinity, 4(2), 111-120. https://doi.org/10.1037/1524-9220.4.2.111

Morry, M. M., \& Staska, S. L. (2001). Magazine exposure: Internalization, selfobjectification, eating attitudes, and body satisfaction in male and female university students. Canadian Journal of Behavioural Science/Revue Canadienne ses Sciences $d u$ Comportement, 33(4), 269-279. https:// doi.org/10.1037/h0087148

Muehlenkamp, J. J., \& Saris-Baglama, R. N. (2002). Self-objectification and its psychological outcomes for college women. Psychology of Women Quarterly, 26(4), 371379. https://doi.org/10.1111/1471-6402. t01-1-00076

Muehlenkamp, J. J., Swanson, J. D., \& Brausch, A. M. (2005). Self-objectification, risk taking, and self-harm in college women. Psychology of Women Quarterly, 29(1), 24-32. https://doi.org/10.1111/ j.1471-6402.2005.00164.x

Murnen, S. K., Smolak, L., Mills, J. A., \& Good, L. (2003) Thin, sexy women and strong, muscular men: grade-school children's responses to objectified images of women and men. Sex Roles, 49(9-10), 427437. https://doi.org/10.1023/A:10258683 20206
Neff, K. D. (2003a). The development and validation of a scale to measure self-compassion. Self and Identity, 2(3), 223-250. https://doi.org/10.1080/15298860309027

Neff, K. D. (2003b). Self-Compassion: An alternative conceptualization of a healthy attitude toward oneself. Self and Identity, 2(2), 85-101. https://doi.org/10.1080/ 15298860309032

Noar, S. M., Morokoff, P. J., \& Redding, C. A. (2002). Sexual assertiveness in heterosexually active men: A test of three samples. AIDS Education and Prevention, 14(4), 330-342. https://doi.org/10.1521/ aeap.14.5.330.23872

Nussbaum, M. C. (1995). Objectification. Philosophy \& Public Affairs, 24(4), 249-291. https://doi.org/10.1111/j.10884963.1995.tb00032.x

Papadaki, E. L. (2007). Sexual objectification: From Kant to contemporary feminism. Contemporary Political Theory, 6(3), 330-348. https://doi.org/10.1057/palgrave. cpt.9300282

Papadaki, E. L. (2010). What is Objectification? Journal of Moral Philosophy, 7(1), 16-36. https://doi.org/10.1163/174046809x 12544019606067

Pisitsungkagarn, K., Taephant, N., \& Attasaranya, P. (2014). Body image satisfaction and self-esteem in Thai female adolescents: the moderating role of self-compassion. International Journal of Adolescent Medicine and Health, 26(3), 333-338. https://doi.org/ 10.1515/ijamh-2013-0307

Pope, H. G., Jr., Olivardia, R., Gruber, A., \& Borowiecki, J. (1999). Evolving ideals of male body image as seen through action toys. International Journal of Eating Disorders, 26, 65-72. https://doi.org/10.1002/ (sici) 1098-108x (199907)26:1<65::aideat8>3.3.co;2-4

Przezdziecki, A., Sherman, K. A., Baillie, A., Taylor, A., Foley, E., \& Stalgis-Bilinski, K. (2012). My changed body: breast cancer, body image, distress and self-compassion. 
Psycho-Oncology, 22(8), 1872-1879. https:// doi.org/10.1002/pon.3230

Quinn, D. M., Kallen, R. W., \& Cathey, C. (2006). Body on my mind: The lingering effect of state self-objectification. Sex Roles, 55(11-12), 869-874. https://doi.org/ 10.1007/s11199-006-9140-x

Quinn, D. M., Kallen, R. W., Twenge, J. M., \& Fredrickson, B. L. (2006). The disruptive effect of self-objectification on performance. Psychology of Women Quarterly, 30(1), 59-64. https://doi.org/ https:// doi.org/10.1111/j.1471-6402.2006.00262.x

Reed, C. L., Stone, V. E., Grubb, J. D., \& McGoldrick, J. E. (2006). Turning configural processing upside down: Part and whole body postures. Journal of Experimental Psychology: Human Perception and Performance, 32, 73-87. https://doi.org/ 10.1037/0096-1523.32.1.73

Roberts, T. A., Calogero, R. M., \& Gervais, S. (2017). Objectification theory: Continuing contributions to feminist psychology. In C. B. Travis, J. W. White, A. Rutherford, W. S. Williams, S. L. Cook, et al. (Eds.). APA handbook of the psychology of women: History, theory, and battlegrounds. 249-271. Washington, DC: American Psychological Association.

Rudman, L. A., \& Borgida, E. (1995). The afterglow of construct accessibility: The behavioral consequences of priming men to view women as sexual objects. Journal of Experimental Social Psychology, 31, 493517. https://doi.org/10.1006/jesp.1995.1022 Rudman, L. A. (1998). Self-promotion as a risk factor for women: The costs and benefits of counterstereotypical impression management. Journal of Personality and Social Psychology, 74(3), 629-645. https:// doi.org/10.1037/0022-3514.74.3.629

Saguy, T., Quinn, D. M., Dovidio, J. F., \& Pratto, F. (2010). Interacting like a body: Objectification can lead women to narrow their presence in social interactions. Psychological Science, 21, 178-182. https:// doi.org/10.1177/0956797609357751
Saul, J. (2003). Feminism: Issues and arguments. Oxford: Oxford University Press.

Shepherd, M., Erchull, M. J., Rosner, A., Taubenberger, L., Forsyth Queen, E., \& McKee, J. (2010). "I'll get that for you": The relationship between benevolent sexism and body self-perceptions. Sex Roles, 64(1-2), 1-8. https://doi.org/10.1007/ s11199-010-9859-2

Sibley, C. G., \& Wilson, M. S. (2004). Differentiating hostile and benevolent sexist attitudes toward positive and negative sexual female subtypes. Sex Roles, 51(1112), 687-696. https://doi.org/10.1007/ s11199-004-0718-x

Steer, A., \& Tiggemann, M. (2008). The role of self-objectification in women's sexual functioning. Journal of Social and Clinical Psychology, 27(3), 205-225. https:// doi.org/10.1521/jscp.2008.27.3.205

Strelan, P., \& Hargreaves, D. (2005). Women who objectify other women: The vicious circle of objectification? Sex Roles, 52(9-10), 707-712. https://doi.org/10.1007/ s11199-005-3737-3

Strelan, P., Mehaffey, S. J., \& Tiggemann, M. (2003). Brief report: Self-objectification and esteem in young women: The mediating role of reasons for exercise. Sex Roles, 48(1-2), 89-95. https://doi.org/10.1023/ a: 1022300930307

Swim, J. K., Hyers, L. L., Cohen, L. L., \& Ferguson, M. J. (2001). Everyday sexism: Evidence for its incidence, nature, and psychological impact from three daily diary studies. Journal of Social Issues, 57(1), 31-53. https://doi.org/10.1111/0022-4537.00200

Szymanski, D. M., Moffitt, L. B., \& Carr, E. R. (2011). Sexual objectification of women: Advances to theory and research. The Counseling Psychologist, 39, 6-38. https://doi.org/10.1177/0011000010378402

Teng, F., Poon, K. T., Zhang, H., Chen, Z., Yang, Y., \& Wang, X. (2016). Situational cuing of materialism triggers self-objectification among women (but not men): The 
Pixellence - 07-07-20 11:48:28 - (c) Humensis

RE0108 U000 - Oasys 19.00x - Page 348 - E1

Revue Annee psychologique 120-3 - Dynamic layout $0 \times 0$

moderating role of self-concept clarity. Personality and Individual Differences, 97, 220-228. https://doi.org/10.1016/j.paid.2016.03.066

Teng, F., You, J., Poon, K.-T., Yang, Y., You, J., \& Jiang, Y. (2017). Materialism predicts young Chinese women's selfobjectification and body surveillance. Sex Roles, 76(7-8), 448-459. https://doi.org/ 10.1007/s11199-016-0671-5

Tiggemann, M., \& Boundy, M. (2008). Effect of environment and appearance compliment on college women's selfobjectification, mood, body shame, and cognitive performance. Psychology of Women Quarterly, 32, 399-405. https:// doi.org/10.1111/j.1471-6402.2008.00453.x

Tiggemann, M., \& Lynch, J. E. (2001). Body image across the life span in adult women: The role of self-objectification. Developmental Psychology, 37(2), 243-253. https://doi.org/10.1037/0012-1649.37.2.243

Tiggemann, M., Martins, Y., \& Kirkbride, A. (2007). Oh to be lean and muscular: Body image ideals in gay and heterosexual men. Psychology of Men \& Masculinity, 8(1), 15-24. https://doi.org/10.1037/15249220.8.1.15

Tiggemann, M., \& Pickering, A. S. (1996). Role of television in adolescent women's body dissatisfaction and drive for thinness. International Journal of Eating Disorders, 20, 199-203. https://doi.org/10.1002/ (sici) 1098-108x(199609)20:2<199::aideat $11>3.0 . \mathrm{co} ; 2-\mathrm{z}$

Tolman, D. L., Impett, E., Tracy, A., \& Michael, A. (2006). Looking good, sounding good: femininity ideology and adolescent girls' mental health. Psychology of Women Quarterly, 30, 85-95. https:// doi.org/10.1111/j.1471-6402.2006.00265.x

Twenge, J. M. (2001). Changes in women's assertiveness in response to status and roles: A cross-temporal meta-analysis, 1931-1993. Journal of personality and social psychology, 81(1), 133-145. https://doi.org/ 10.1037/0022-3514.81.1.133
Vaes, J., Paladino, M. P., \& Puvia, E. (2011). Are sexualised women complete human beings? Why men and women dehumanise sexually objectified women. European Journal of Social Psychology, 41, 774-785. https://doi.org/10.1002/ejsp.824

Vigarello G. (2004). Histoire de la beauté. Le corps et l'art d'embellir de la Renaissance à nos jours. Paris : Le Seuil.

Von Feigenblatt, O. F. (2010). A brief analysis of popular aesthetics in Thailand: Subjective culture vs. objective culture. Journal of Social Sciences, 22(1), 61-63. https://doi.org/10.1080/09718923.2010.11 892785

Ward, L. M. (2016). Media and sexualization: State of empirical research, 19952015. Journal of Sex Research, 53, 560-577. http://dx.doi.org/10.1080/ 00224499.2016 .1142496

Wolf, N. (1991). The Beauty Myth: How Images of Beauty Are Used Against Women. New York: William Morrow \& Company.

Wollast, R. (2018). La compassion pour soi-même peut-elle protéger des conséquences néfastes liées à une image corporelle négative? Sextant, 35, 117-128.

Wollast, R., Coertjens, L., Bernard, P., \& Klein, O. (soumis). Is the Relation between Sexism and Body Image Culture-Dependent? A Comparison between Belgium and Thailand. Manuscrit en révision.

Wollast, R., Puvia, E., Bernard, P., Tevichapong, P., \& Klein, O. (2018). How sexual objectification generates dehumanization in Western and Eastern Cultures-a comparison between Belgium and Thailand. Swiss Journal of Psychology, 77(2), 69-82. https://doi.org/10.1024/1421-0185/a000209 Wollast, R., Riemer, A., Bernard, P., Leys, C., Kotsou, I., \& Klein, O. (2019). How self-compassion moderates the effect of body surveillance on subjective happiness and depression among Women. Scandinavian Journal of Psychology. https://doi.org/ 10.1111/sjop.12553 
Pixellence - 07-07-20 11:48:28 - (c) Humensis

RE0108 U000 - Oasys 19.00x - Page 349 - E

Revue Annee psychologique 120-3 - Dynamic layout $0 \times 0$

Wollast, R., Riemer, A., Gervais, S., Grigoryan, L., Bernard, P., \& Klein, O. (soumis). How self-compassion moderates the effect of body surveillance on body shame for women from America, Belgium, Russia, and Thailand. Manuscrit en révision.

Wollast, R., Riemer, A., Sarda, E., \& Klein, O. (soumis). How self-compassion moderates the relation between body surveillance on body shame among men and women. Manuscrit en révision.

Yager, Z., \& O’Dea, J. A. (2008). Prevention programs for body image and eating disorders on University campuses: a review of large, controlled interventions. Health Promotion International, 23, 173-189. https:/doi.org/10.1093/heapro/dan004 